\title{
Absolute emission rates of Spontaneous Parametric Down Conversion into single transverse Gaussian modes
}

\author{
Alexander Ling ${ }^{1,2}$, Antía Lamas-Linares ${ }^{2}$ and Christian Kurtsiefer ${ }^{2}$ \\ ${ }^{1}$ Temasek Labs, National University of Singapore, Singapore 117508 and \\ ${ }^{2}$ Centre for Quantum Technologies / Department of Physics, \\ National University of Singapore, Singapore 11754 * *
}

(Dated: October 25, 2018)

\begin{abstract}
We provide an estimate on the absolute values of the emission rate of photon pairs produced by spontaneous parametric down conversion in a bulk crystal when all interacting fields are in single transverse Gaussian modes. Both collinear and non-collinear configurations are covered, and we arrive at a fully analytical expression for the collinear case. Our results agree reasonably well with values found in typical experiments, which allows this model to be used for understanding the dependency on the relevant experimental parameters.
\end{abstract}

\section{INTRODUCTION}

For the last two decades, spontaneous parametric down-conversion (SPDC) has been the workhorse process for the generation of correlated photon pairs. These can easily be cast into maximally entangled states, which are useful e.g. for violating Bell inequalities [1], or for the investigation of other fundamental aspects of quantum mechanics. Progress in SPDC-based photon pair sources has allowed the emitted pairs from such sources to play a leading role in demonstration of quantum information techniques, and made its way to almost practical applications like quantum key distribution.

A number of studies have established the basic understanding of SPDC [2, 3, 4] , based on energy conservation, and momentum conservation when participating light fields are treated as plane waves [5].

Many of the more recent applications often necessitate significant manipulation and transport of the photon pairs; this can be achieved in a convenient way by guiding the light in single mode optical fibers. The basic idea of modeling SPDC in this regime is to map the optical modes propagating in the fibers into freely propagating modes of the electromagnetic field in the nonlinear conversion material, where they interact with a pump field. These freely propagating spatial modes can be described in good approximation by paraxial Gaussian beams, and any optimization strategy will involve some sort of mode matching of such interacting beams.

Previous studies of SPDC light coupled into single mode fibers have focused in optimizing the coupling efficiency, defined as the ratio of photon pairs to single photons that are observed because this is a quantity which can be measured easily in an experiment. This quantity is important for developing loophole-free tests of Bell's inequality [ 6 , heralded single photon sources $[7,6$, , 9], or simply sources of high pair brightness [10].

So far, theoretical work in this area has focused mostly in such secondary parameters, and no closed expression for the absolute rate of photon pairs was available for typ- ical experimental configurations. This made it difficult to estimate whether a particular experimental source implementation could be improved with respect to a particular figure-of-merit, be it total rate or spectral brightness.

In this paper, we try to address this problem and derive an expression for the absolute rate of SPDC emission from a bulk crystal into Gaussian modes. The work connects to earlier investigations of absolute SPDC rates with beams of finite diameter by Kleinman and Klyshko 3, 4]. It was found there that the overall rate of pair production is independent of the spot-size of the pump beam [3] , and that the conversion efficiency of pump photons into correlated pairs integrated over all emission directions [4] is in the order of $10^{-8}$ per $\mathrm{mm}$ for a typical non-linear material. The restriction on specific spatial modes defined by single mode optical fibers in the more recent applications, however, made it difficult to relate their results directly to experiments. Our description applies both to Type I and II phase matching conditions, and covers collinear and non-collinear geometries important for the generation of polarization-entangled photon pairs [11, 12].

\section{MODEL}

The basic process of SPDC can be understood as the spontaneous decay of a photon from a pump field into two daughter photons propagating in two - possibly different - target modes, with the process being mediated by a material with a nonlinear optical susceptibility. The physical implementation of SPDC utilizes the lowest order of the nonlinear susceptibility tensor $\chi^{(2)}$ in an appropriate material. While energy conservation allows the decay process to take place in many target modes, phase matching requirements need to be engineered to allow conversion to take place into any particular pair of directions.

The physical model of the three interacting optical modes is depicted in figure 1. We treat the pump beam and the target modes for the down-converted light as 


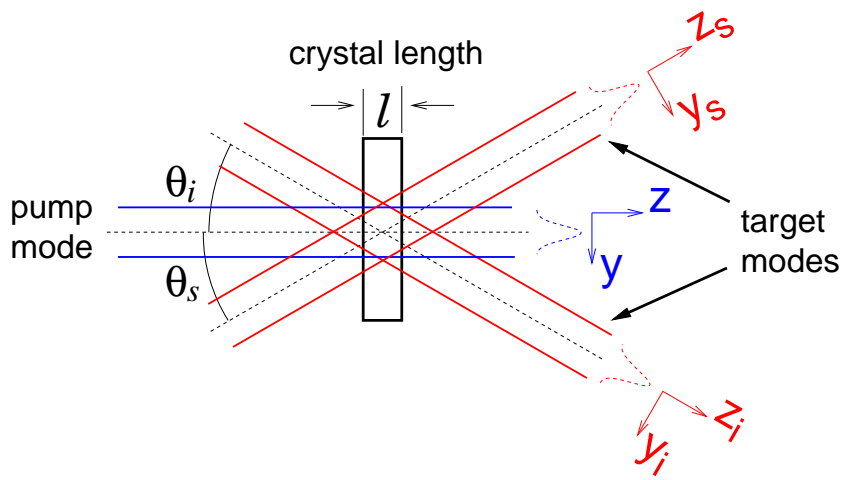

FIG. 1: (Color online) Schematic of the down conversion model considered in this paper. Pump, signal and idler beams are treated as paraxial beams with a Gaussian transverse mode. The $\mathrm{x}$-axis is perpendicular to the plane of the diagram. Coordinate systems of the signal and idler $\left(x_{s, i}, y_{s, i}, z_{s, i}\right)$ are tilted by angles $\theta_{s}, \theta_{i}$ with respect to the coordinate system of the pump.

propagating paraxial beams with a Gaussian transverse profile. The beams overlap within a nonlinear optical crystal of finite length $l$, with surfaces normal to the propagation direction of the pump beam. Pump and target modes propagate in one plane, but need not to be parallel. We further assume that the three interacting modes overlap in a region without a significant variation of the transverse profile along their respective propagation directions. This is a reasonable assumption for typical Gaussian beam parameters and conversion crystal lengths used in experiments [10].

We follow the tradition of referring to the target modes as signal (index $s$ ) and idler (index $i$ ) and choose coordinate systems where the $z_{s, i, p}$ directions are parallel to the main propagation direction for each mode $s, i, p(p$ refers to the pump mode). The spatial mode function of the electrical field for each of the modes can be written as

$$
g(\mathbf{r})=e^{i k z} \cdot U(x, y)=e^{i k z} \cdot e^{-\frac{x^{2}+y^{2}}{W^{2}}},
$$

where $k$ denotes the $z$-component of the corresponding wave vector, $W$ the Gaussian beam waist parameter, and $x, y, z$ refer to the corresponding coordinate system for each mode. To simplify the overlap calculations, we use normalization constants $\alpha$ for the envelope functions $U(x, y)$ such that

$$
\alpha^{2} \int d x d y|U(x, y)|^{2}=1
$$

in their corresponding coordinate systems, which implies

$$
\alpha_{p, s, i}=\sqrt{\frac{2}{\pi W_{p, s, i}^{2}}} .
$$

We note that the spatial mode function $g(\mathbf{r})$ fulfills Maxwell's equations only approximately. For the calcu- lations presented below, however, this poses no problem. Furthermore, the dispersion relation connected with this mode function has the confinement correction:

$$
\omega^{2}=c^{2}\left(k^{2}+\frac{2}{W^{2}}\right)
$$

Again, for practical beam diameters $W$ of about 100 wavelengths considered in this paper, this correction term is small enough to safely neglect it.

\section{Pump mode}

The pump mode is aligned with the main coordinate system $x, y, z$, and treated as a classical monochromatic field of amplitude $E_{p}^{0}$. We further assume (as is customary) that we have no significant depletion of the pump in the downconversion crystal. The electrical field of the pump can thus be written as

$$
\begin{aligned}
\mathbf{E}_{p}(\mathbf{r}, t) & =\frac{1}{2}\left[\mathbf{E}_{p}^{(+)}(\mathbf{r}, t)+\mathbf{E}_{p}^{(-)}(\mathbf{r}, t)\right] \\
& =\frac{1}{2}\left[E_{p}^{0} \mathbf{e}_{p} g_{p}(\mathbf{r}) e^{-i \omega_{p} t}+c . c .\right]
\end{aligned}
$$

with a polarization vector $\mathbf{e}_{p}$, and a corresponding angular frequency $\omega_{p}$. Using the normalization expression (2), we can connect the electrical field amplitude $E_{p}^{0}$ with the optical power $P$ in the pump beam:

$$
\left|E_{p}^{0}\right|^{2}=\alpha_{p}^{2} \frac{2 P}{\epsilon_{0} n_{p} c}
$$

with the refractive index $n_{p}$ for the pump field, the electrical field constant $\epsilon_{0}$ and the speed of light $c$ in vacuum.

\section{Target modes}

First, we need to take care of the possibly non-collinear propagation of the target modes with respect to the pump. By introducing target mode angles $\theta_{s, i}$, and using an orientation as indicated in figure 1, we express the spatial coordinates of the target modes in terms of the main coordinates $x, y, z$ :

$$
\left(\begin{array}{l}
x_{s, i} \\
y_{s, i} \\
z_{s, i}
\end{array}\right)=\left(\begin{array}{ccc}
1 & 0 & 0 \\
0 & \cos \theta_{s, i} & \pm \sin \theta_{s, i} \\
0 & \mp \sin \theta_{s, i} & \cos \theta_{s, i}
\end{array}\right)\left(\begin{array}{l}
x \\
y \\
z
\end{array}\right)
$$

To arrive at a rate of photon pairs generated via SPDC, we use the quantized field operators. We do that by introducing a quantization length $L$ in propagation direction for clarity in the counting of modes, and postulate periodic boundary conditions; later we will drop this requirement. Following the notation in equation (5), the 
electrical field operators take the form

$$
\begin{aligned}
\hat{\mathbf{E}}_{s, i}= & \frac{1}{2}\left[\hat{\mathbf{E}}_{s, i}^{(+)}(\mathbf{r}, t)+\hat{\mathbf{E}}_{s, i}^{(-)}(\mathbf{r}, t)\right] \\
= & \frac{i}{2} \sum_{k_{s, i}} \sqrt{\frac{2 \hbar \omega_{s, i}}{n_{s, i}^{2} \epsilon_{0}}} \frac{\alpha_{s, i}}{\sqrt{L}} \mathbf{e}_{s, i} g_{s, i}(\mathbf{r}) e^{-i \omega_{s, i} t} \hat{a}_{k_{s, i}} \\
& + \text { h.c. }
\end{aligned}
$$

Here, $\mathbf{e}_{s, i}$ indicate the polarization vectors, and $n_{s, i}$ and $\omega_{s, i}$ the corresponding refractive indices and angular frequencies of the target modes. The modes are indexed by the scalar moduli $k_{s, i}$, and the corresponding full wave vectors in the pump coordinates are given by

$$
\mathbf{k}_{s, i}=k_{s, i}\left(\mp \sin \theta_{s, i} \mathbf{e}_{y}+\cos \theta_{s, i} \mathbf{e}_{z}\right) .
$$

The longitudinal wave vector components serve as a complete discrete mode index $k_{s, i}=2 \pi m_{s, i} / L$ with integer numbers $m_{s, i}$. The coefficients before the raising and lowering operators in eq. (8) are chosen such that the free field Hamiltonian $\hat{H}_{0}$ for the target modes takes the usual form

$$
\hat{H}_{0}=\sum_{k_{s, i}} \hbar \omega_{s, i}\left(\hat{a}_{k_{s, i}}^{\dagger} \hat{a}_{k_{s, i}}+\frac{1}{2}\right) .
$$

\section{Interaction Hamiltonian}

The SPDC process is enabled by a nonlinear optical material whose presence is described by the Hamiltonian $\hat{H}_{I}$, written in the interaction picture with time dependence of the raising and lowering operators [13]:

$$
\begin{aligned}
\hat{H}_{I}= & -\frac{2 \epsilon_{0} \chi^{(2)}}{8} \int_{-\infty}^{\infty} d x d y \int_{-l / 2}^{l / 2} d z \mathbf{E}_{p}^{(+)} \hat{\mathbf{E}}_{s}^{(-)} \hat{\mathbf{E}}_{i}^{(-)}+h . c . \\
= & d \int_{-\infty}^{\infty} d x d y \int_{-l / 2}^{l / 2} d z \sum_{k_{s}, k_{i}} \frac{\hbar \sqrt{\omega_{i} \omega_{s}}}{n_{s} n_{i}} \frac{\alpha_{s} \alpha_{i} E_{p}^{0}}{L} \times \\
& \times e^{-i \Delta \omega t} g_{p}(\mathbf{r}) g_{s}^{*}(\mathbf{r}) g_{i}^{*}(\mathbf{r}) \hat{a}_{k_{s}}^{\dagger}(t) \hat{a}_{k_{i}}^{\dagger}(t)+\text { h.c. }(11)
\end{aligned}
$$

We have assumed a crystal of infinite transverse $(x, y)$ extent, which is justified when the beam diameters are much smaller than the crystal dimensions. We introduce a frequency mismatch $\Delta \omega=\omega_{p}-\omega_{s}-\omega_{i}$. The effective non-linearity $d$ captures the contraction of the nonlinear susceptibility tensor with the corresponding polarization vectors $\left(2 d=\mathbf{e}_{p} \chi^{(2)}: \mathbf{e}_{s} \mathbf{e}_{i}\right)$ [13]. With this notation, the type of phase matching condition (type I or II) is reflected in an appropriate effective non-linearity $d$.

Most of the scaling aspects of the parametric down conversion process connected with the geometry of the interaction are determined by the overlap integral $\Phi(\Delta k)$ of the three mode functions $g_{p, s, i}(\mathbf{r})$ in the crystal:

$$
\begin{aligned}
\Phi(\Delta \mathbf{k}) & =\int d z \int d y d x g_{p}(\mathbf{r}) g_{s}^{*}(\mathbf{r}) g_{i}^{*}(\mathbf{r}) \\
& =\int d z \int d y d x e^{i \Delta \mathbf{k} \cdot \mathbf{r}} U_{p}(\mathbf{r}) U_{s}(\mathbf{r}) U_{i}(\mathbf{r}) .
\end{aligned}
$$

In this expression, $\Delta \mathbf{k}=\mathbf{k}_{p}-\mathbf{k}_{s}-\mathbf{k}_{i}$ describes the wave vector mismatch. Since pump and target modes are defined in the $y$ - $z$ plane, there are no wave vector components in the $x$-direction and hence $\Delta k_{x}=0$. Carrying out the integration in the transverse directions $(x, y)$ we arrive at

$$
\Phi(\Delta \mathbf{k})=\frac{\pi}{\sqrt{A \cdot C}} e^{-\frac{\Delta k_{y}^{2}}{4 C}} \int d z e^{-H z^{2}+i z K},
$$

with the abbreviations

$$
\begin{aligned}
& A=\frac{1}{W_{p}^{2}}+\frac{1}{W_{s}^{2}}+\frac{1}{W_{i}^{2}} \\
& C=\frac{1}{W_{p}^{2}}+\frac{\cos ^{2} \theta_{s}}{W_{s}^{2}}+\frac{\cos ^{2} \theta_{i}}{W_{i}^{2}} \\
& D=\frac{\sin 2 \theta_{s}}{W_{s}^{2}}-\frac{\sin 2 \theta_{i}}{W_{i}^{2}} \\
& F=\frac{\sin ^{2} \theta_{s}}{W_{s}^{2}}+\frac{\sin ^{2} \theta_{i}}{W_{i}^{2}} \\
& H=F-\frac{D^{2}}{4 C} \\
& K=\Delta k_{y} \frac{D}{2 C}+\Delta k_{z}
\end{aligned}
$$

The exponential term before the residual integral in eq. (13) represents the approximate transverse wave vector mismatch. This term can be ignored only if one of the beams is infinitely large $\left(W_{p, s, i} \rightarrow \infty\right)$, or if there is perfect transverse phase matching.

The residual integral along $z$ in (13) can be re-written in a form that allows for a physical interpretation. We introduce $\Phi_{z}$ where

$$
\begin{aligned}
\Phi_{z} & :=\int_{-l / 2}^{l / 2} d z e^{-H z^{2}+i z K} \\
& =l \cdot \int_{0}^{1} d u e^{-\Xi^{2} u^{2}} \cos (\Delta \varphi u) .
\end{aligned}
$$

The phase mismatch is now defined as $\Delta \varphi:=K l / 2$. The argument $\Xi:=\sqrt{H} l / 2$ in the exponential can be viewed as a "walk-off" parameter due to non-collinear mode propagation. This parameter is useful for identifying a thin crystal and a thick crystal regime [6]. In our model, these regimes refer to the physical boundary conditions imposed on the interaction volume by the geometry of the pump and target modes. 


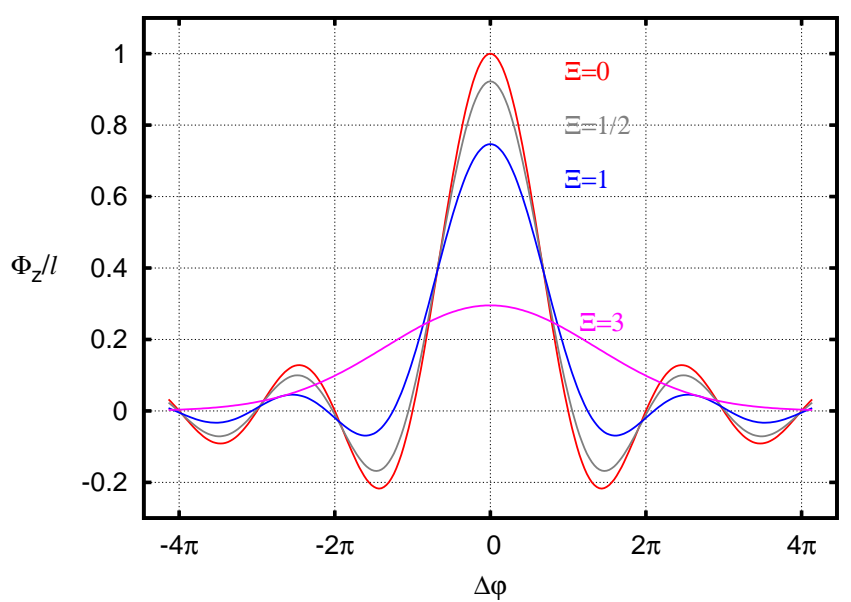

FIG. 2: (Color online) Longitudinal overlap function $\Phi_{z} / l$ as a function of the total phase mismatch $\Delta \varphi=K l / 2$ over the crystal for various walk-off parameters $\Xi$. For $\Xi=0$, the typical sinc shaped spectral distribution is revealed, whereas for large walk-off parameters $\Xi>1$ the phase matching condition is determined by the overlap region formed by pumpand target modes, and develops into a Gaussian distribution.

In the thick crystal regime with a large walk-off parameter $(\Xi>1)$, the overlap integral $\Phi_{z}$ depends mostly on the characteristic beam parameters $W_{p, s, i}$ and not much on the physical boundaries of the non-linear material. For $\Xi \rightarrow \infty$ the length of the crystal ceases to play a role altogether:

$$
\begin{aligned}
\Phi_{z} & \approx l \frac{\sqrt{\pi}}{2 \Xi} \operatorname{Erf}(\Xi) \\
& =\sqrt{\frac{\pi}{H}} \operatorname{Erf}(\Xi)
\end{aligned}
$$

The thin crystal regime refers to a small walk-off parameter, $\Xi \ll 1$, so that the characteristic beam parameters have almost no influence on $\Phi_{z}$. In particular, this applies for collinear arrangement of all modes $\left(\theta_{i}=\theta_{s}=0\right)$, where $\Xi=0$. In this case, $K=\Delta k_{z}$, and

$$
\Phi_{z}=l \operatorname{sinc}(\Delta \varphi)
$$

This reveals the well-known influence of the longitudinal phase mismatch on the downconversion spectral properties [3]. Figure 2] shows the overlap contribution $\Phi_{z} / l$ as a function of the phase mismatch $\Delta \varphi$ for various walk-off parameters $\Xi$. We note that as $\Xi$ becomes large, the spectrum becomes Gaussian-like. If $\Xi$ is identified as the degree of (non)collinearity, it suggests that the SPDC spectral profile far from collinear emission can be very broad. In other words, for the same beam parameters $W_{s, i, p}$, the bandwidth of collinear emission will be narrower than for non-collinear emission.

\section{Spectral Emission Rate}

To obtain absolute emission rates, we make use of Fermi's Golden Rule as an expression for the transition rate $R\left(k_{s}\right)$ between the initial vacuum state $|i\rangle=$ $\left|0_{k_{s}}, 0_{k_{i}}\right\rangle$, and a final state $|f\rangle=\hat{a}_{k_{s}}^{\dagger} \hat{a}_{k_{i}}^{\dagger}\left|0_{k_{s}}, 0_{k_{i}}\right\rangle$ with the mode pair $k_{s}, k_{i}$ populated with one photon each. Fermi's rule applies for asymptotic scattering rates, so the relation between $k_{s}$ and $k_{i}$ is fixed by energy conservation:

$$
\Delta \omega=\omega_{p}-k_{s} \frac{c}{n_{s}}-k_{i} \frac{c}{n_{i}}=0
$$

We first evaluate the transition rate $R\left(k_{s}\right)$ to a fixed signal target mode $k_{s}$. The density of states $\rho$ per unit of energy $\Delta E=\hbar \Delta \omega$ is extracted out of a quasi-continuum of states for the mode $k_{i}$ :

$$
\rho(\Delta E)=\frac{\Delta m}{\Delta k_{i}} \frac{\partial k_{i}}{\partial(\hbar \Delta \omega)}=\frac{L}{2 \pi} \frac{n_{i}}{\hbar c},
$$

where $\Delta m / \Delta k_{i}=L / 2 \pi$ denotes the number of modes per unit of wave vector component $k_{i}$.

With the transition matrix element expressed in terms of the overlap integral $\Phi(\Delta \mathbf{k})$,

$$
\left\langle f\left|\hat{H}_{I}\right| i\right\rangle=d \frac{\hbar \sqrt{\omega_{s} \omega_{i}}}{n_{s} n_{i}} \frac{\alpha_{s} \alpha_{i}}{L} E_{p}^{0} \Phi(\Delta \mathbf{k}),,
$$

the transition rate is then given by

$$
\begin{aligned}
R\left(k_{s}\right) & =\frac{2 \pi}{\hbar}\left|\left\langle f\left|\hat{H}_{I}\right| i\right\rangle\right|^{2} \rho(\Delta E) \\
& =\left|d \alpha_{s} \alpha_{i} E_{p}^{0} \Phi(\Delta \mathbf{k})\right|^{2} \frac{\omega_{s} \omega_{i}}{n_{s}^{2} n_{i} c L}
\end{aligned}
$$

The spectral emission rate per unit of angular frequency $\omega_{s}$ is obtained by multiplying $R\left(k_{s}\right)$ with the number of modes $k_{s}$ in a unit interval of $\omega_{s}$, which is $L n_{s} / 2 \pi c$. We finally arrive at

$$
\frac{d R\left(\omega_{s}\right)}{d \omega_{s}}=\left[\frac{d \alpha_{s} \alpha_{i} E_{p}^{0} \Phi(\Delta \mathbf{k})}{c}\right]^{2} \frac{\omega_{s} \omega_{i}}{2 \pi n_{s} n_{i}}
$$

At this point, the earlier introduced quantization length $L$ has vanished as expected.

\section{Total Emission Rate}

We now can determine the total pair generation rate by integrating the spectral rate density over all frequencies $\omega_{s}$. Assuming that the overlap $\Phi(\Delta \mathbf{k})$ is only nonvanishing over a small range of frequencies $\omega_{s}$, the total pair generation rate can be written as

$$
R_{T}=\left[\frac{d \alpha_{s} \alpha_{i} E_{p}^{0}}{c}\right]^{2} \frac{\omega_{s} \omega_{i}}{2 \pi n_{s} n_{i}} \int d \omega_{s}|\Phi(\Delta \mathbf{k})|^{2}
$$


The dependency of $\Phi(\Delta \mathbf{k})$ on $\omega_{s}$ can be quite involved, as in the non-collinear case $\left(\theta_{i, s} \neq 0\right)$ both $\Delta k_{y}\left(\omega_{s}\right)$ and $\Delta k_{z}\left(\omega_{s}\right)$ must be considered. However, the alignment criteria for most experimental setups assume perfect longitudinal phase matching to arrive at the collection angles for degenerate downconversion [10, 11]. In these collection directions, the target mode angles $\theta_{s, i}$ are equal. Furthermore, the typical experiments use identical collection mode diameters for signal and idler, $W_{s}=W_{i}$ [10]. Under these two conditions, the phase mismatch $\Delta \varphi$ depends only on $\Delta k_{z}$.

We now consider the exponential term for the overlap $\Phi$ in eq. (13) that contains $\Delta k_{y}$. For experiments where light centered on the degenerate wavelengths with a small bandwidth is collected $(\approx 2 \mathrm{~nm}$ on either side of the center [10]), we will assume perfect transverse phase matching. A complete treatment with non-zero transverse phase mismatch requires a numerical procedure [14].

With perfect transverse phase matching, we can carry out the integration in eq. (30) by re-parameterizing the frequencies of the signal and idler about the degenerate SPDC frequency: $\omega_{s}=\frac{\omega_{p}}{2}-\delta_{\omega}$ and $\omega_{i}=\frac{\omega_{p}}{2}+\delta_{\omega}$. We approximate $\omega_{s} \omega_{i} \approx \frac{\omega_{p}^{2}}{4}$ by ignoring terms $O\left(\delta_{w}^{2}\right)$. From energy conservation eq. (24) and the phase matching condition

$$
\Delta k_{z}=n_{s} \omega_{s} \cos \theta_{s}+n_{i} \omega_{i} \cos \theta_{i}-n_{p} \omega_{p}
$$

we obtain a dispersion relation between $d \omega_{s}$ and $d\left(\Delta k_{z}\right)$ :

$$
d\left(\Delta k_{z}\right)=\frac{\left(n_{i} \cos \theta_{i}-n_{s} \cos \theta_{s}\right)}{c} d \omega_{s}
$$

The emission rate can now be integrated over the longitudinal wave vector mismatch $\Delta k_{z}$,

$$
R_{T}=\frac{d^{2}\left(\alpha_{i} \alpha_{s} E_{p}^{0}\right)^{2} \omega_{p}^{2}}{4 c n_{s} n_{i}(2 \pi)\left(n_{i} \cos \theta_{i}-n_{s} \cos \theta_{s}\right)} \int|\Phi(\Delta \mathbf{k})|^{2} d\left(\Delta k_{z}\right) .
$$

Effectively, this is the pair emission rate for all allowed wavelengths in the direction defined by our paraxial beams. If we recall that the pump has a Gaussian envelope, and choose all beam characteristics to be equal $\left(W_{p}=W_{s}=W_{i}\right)$, the rate $R_{T}$ finally can be written as

$$
\begin{aligned}
R_{T}= & \frac{4 d^{2} P l \omega_{p}^{2}}{3 \pi n_{p} n_{s} n_{i} \epsilon_{0} c^{2}\left(\pi W_{p}^{2}\right)\left(1+\cos \theta_{i}^{2}+\cos \theta_{s}^{2}\right)} \times \\
& \times \frac{1}{\left(n_{i} \cos \theta_{i}-n_{s} \cos \theta_{s}\right)} S
\end{aligned}
$$

with the spectral integral $S:=\int\left|\frac{\Phi_{z}\left(\Delta \mathbf{k}_{\mathbf{z}}\right)}{l}\right|^{2} d\left(\Delta k_{z} l / 2\right)$. The absolute emission rate is proportional to $S$, which has a dependence on the value of the walk-off parameter $\Xi$ as shown in figure 3. The spectral integral assumes its largest value $S=\pi$ in the thin crystal limit. In this

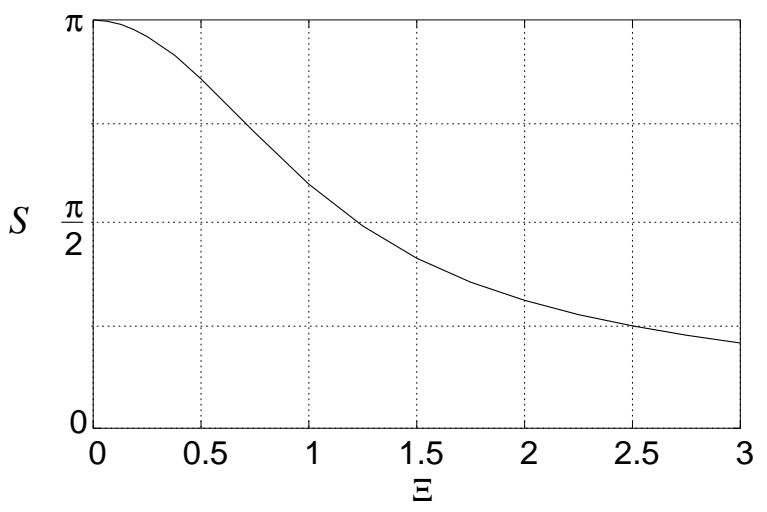

FIG. 3: Variation of the spectral integral $S$ with walk-off parameter $\Xi$. Since the absolute emission rate is proportional to $S$, the largest absolute rate is obtained in the thin-crystal limit with $\Xi=0$.

limit, closed form expressions for the spectral and total rates are

$$
\begin{aligned}
\frac{d \tilde{R}\left(\omega_{s}\right)}{d \omega_{s}} & =\frac{2 d^{2} \omega_{p}^{2} P l^{2} \operatorname{sinc}^{2}\left(\Delta k_{z} l / 2\right)}{9 \pi n_{p} n_{s} n_{i} \epsilon_{0} c^{3}\left(\pi W_{p}^{2}\right)} \text { and } \\
\tilde{R}_{T} & =\frac{4 d^{2} P l \omega_{p}^{2}}{9 n_{s} n_{i} n_{p} \epsilon_{0} \pi W_{p}^{2}\left(n_{i}-n_{s}\right) c^{2}} .
\end{aligned}
$$

\section{Dependence of Emission Rate on Beam Waists}

Although it is convenient to set all beam waists to be equal, this is not necessary. In fact, it can be shown that this choice does not maximize the total emission rate for a given optical pump power. Carrying out the more general derivation to arrive at an expression similar to (36), the dependency on the various beam waists $W_{p}, W_{s}$ and $W_{i}$ (again in the thin crystal limit) can be written as

$$
R_{T} \propto \frac{1}{W_{p}^{2} W_{s}^{2} W_{i}^{2}\left(\frac{1}{W_{p}^{2}}+\frac{1}{W_{s}^{2}}+\frac{1}{W_{i}^{2}}\right)^{2}} .
$$

To develop an alignment strategy, we may assume that the collection modes are identical $\left(W_{s}=W_{i}=W\right)$, but we re-express the pump waist as $W_{p}=\gamma W$, so we obtain

$$
\tilde{R}_{T} \propto R_{T} \propto \frac{1}{W^{2}\left(\frac{1}{\gamma}+2 \gamma\right)^{2}} .
$$

This relationship is illustrated in figure 4 and exhibits a maximum of $\tilde{R}_{T}$ for $\gamma=\frac{1}{\sqrt{2}}$. For $\gamma=1$, the emission rate is about $12 \%$ lower than the maximum value. This suggests that experimental setups that are designed with equal beam waists for pump and collection modes may be further optimized, and the simple argument of maximizing a mode overlap [10] with matching beam waists does not hold. 


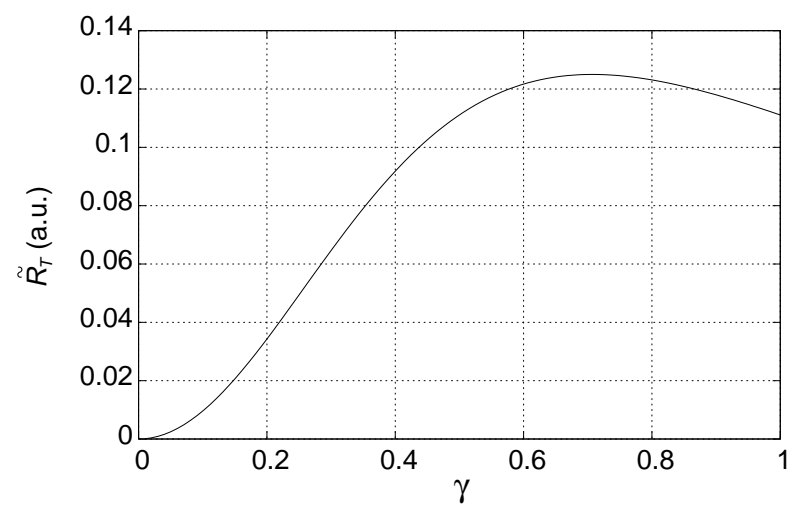

FIG. 4: Dependence of the total pair rate $\tilde{R}_{T}$ on the ratio $\gamma$ between target and pump waist. The maximum emission rate can be expected at $\gamma=1 / \sqrt{2}$.

\section{PHYSICAL INTERPRETATION AND COMPARISON TO EXPERIMENTS}

While $R_{T}$ is proportional to crystal length $l$, the spectral rate $d R\left(\omega_{s}\right) / d \omega_{s}$ is proportional to the square of a sinc function. This is in agreement with results from previous work [3]. However, our expression reveals dependencies on other factors, namely emission geometry and pump spot-size. The expression for $R_{T}$ reveals that the emission rate is higher in a collinear geometry compared to a non-collinear case. This can be intuitively understood because the non-collinear case has a smaller interaction volume.

We find that the absolute rate $R_{T}$ is proportional to the square of pump frequency since we re-parameterize the signal and idler about the degenerate frequency, so downconversion efficiency can be improved with shorter wavelength pumps. Both spectral and total emission rates are inversely proportional to the mode area of the beams, in contrast to previous studies which showed that the total SPDC cannot be enhanced by focusing [3, 15]. There, however, SPDC emission was not considered for specific transverse modes. The dependence of emission rates on the mode area have been reported in a previous analysis of SPDC in waveguide structures [16]. This is not surprising because the emission into paraxial beams is essentially the same problem as SPDC in waveguides, where the target modes are quantized in one dimension only. We note that our equation (35) is similar to the equation obtained in reference [16].

We should not draw the conclusion, however, that SPDC emission into single transverse modes can be arbitrarily enhanced by tight focusing. Our model is only valid in cases where the transverse profile of the beams does not vary significantly over the crystal length. For an optimization study of focus size on SPDC emission we refer the reader to $[9]$.

For explicit comparison of eq. (34) with experimental values, we consider our experimental setup (similar to that used in reference [17]). In this experiment a pump beam (beam waist, $W_{p}=82 \mu \mathrm{m}$ ) at a wavelength of 351.1 $\mathrm{nm}$ is incident on a $2 \mathrm{~mm}$ thick $\beta$-Barium Borate (BBO) crystal. Two single mode fibers are used to collect degenerate downconverted photons, which is estimated to have an external emission angle of $3.1^{\circ}$. The collection modes also have beam waists of $W_{s, i}=82 \mu \mathrm{m}$.

Specifically for BBO the effective non-linearity is given by $d=d_{22} \cos ^{2} \theta_{c} \cos 3 \phi_{c}$. The angle between pump wave vector and crystal optical axis is $\theta_{c}=49.7^{\circ}$, while the azimuthal angle is $\phi_{c}=60^{\circ}$, resulting in an effective nonlinearity of $9 \times 10^{-13} \mathrm{~m} / \mathrm{V}\left(d_{22}=2.11 \times 10^{-12} \mathrm{~m}^{-1} \mathrm{~V}^{-1}\right.$ according to [18]). The observed pair rate is approximately 800 pairs $\mathrm{mW}^{-1} \mathrm{~s}^{-1}$ with a pair-to-singles ratio of 0.23 .

The walk-off parameter for this setup is $\Xi=0.933$, indicating that the overlap integral is intermediate between the thin and thick crystal limits. The largest observable rate according to our model is $2\left(0.23 \times R_{T}\right)=$ $1100 \mathrm{~mW}^{-1} \mathrm{~s}^{-1}$. The additional factor of 2 is used because in experiments, the geometry is used to collect downconversion emission in two decay paths.

The source of the discrepancy between experiment and our model is hard to identify. The assumptions used in the model make it an overestimate, primarily in the reparameterizing of signal and idler frequencies about the degenerate wavelength. Experimentally, there are several sources of uncertainty, the main one being the difficulty in establishing pump power very accurately. For example, the average observed value was arrived by measuring the power using two different power-meters (a Newport Model 818-UV reported $11.7 \mathrm{~mW}$ while a Coherent Fieldmaster reported $9 \mathrm{~mW}$ ). The uncertainty in pump power estimation, however, is not sufficient to make the observed result compatible with the calculated value.

According to the model, the conversion efficiency into Gaussian transverse modes for our experimental setting will be $3 \times 10^{-12} \mathrm{~mm}^{-1}$ of crystal length. Other experimentally reported rates in the literature reveal similar downconversion efficiencies [8, 10, 19]. The total conversion efficiency of SPDC was found to be on the order of $3 \times 10^{-8} \mathrm{~mm}^{-1} \mathrm{sr}^{-1}$ by Klyshko [4] (for degenerate SPDC with a $500 \mathrm{~nm}$ pump wavelength). Experimentally, our collection angle is $3.3 \times 10^{-5}$ sr. If we convert our units to be comparable with Klyshko's result, we obtain an efficiency of $7 \times 10^{-8} \mathrm{~mm}^{-1} \mathrm{sr}^{-1}$.

\section{CONCLUSION}

In conclusion, we have presented expressions that provide absolute values for the rate and bandwidth of correlated pairs emitted in bulk crystal SPDC which are in a single Gaussian mode. These modes may be defined by the collection profile of single mode fibers, selecting 
emission in a specific pair of directions. The single mode treatment reduces the complexity in the final expression for the rate equations.

We find that the expression for absolute rates given by the model are slightly larger than experimental observations. The model may thus be treated as an idealized case for the total pair emission rate. The small difference between experimentally observed rates and predictions according to the closed expression for $R_{T}$, however, suggests that experimental setups using single mode collection fibers (e.g. [8, 10]) operate close to the optimal limit.

Substantial increase of the emission rates are to be expected from larger non-linearities, since emission rates are proportional to $d^{2}$. Small mode diameters are also expected to enhance emission rates, as has been convincingly reported for SPDC experiments using waveguide structures [20, 21, 22], and a similar theoretical analysis [16] for those cases. Overall spectral brightness will be improved by combining larger non-linearities with collinear mode confinement in longer structures. Even then, however, the spectral width is still ultimately determined by the longitudinal wave vector mismatch. This indicates that very dramatic improvements (by several orders of magnitude) to the generated pair rate in a narrow bandwidth necessary for addressing atomic systems is not very likely to be expected from bulk crystal emission.

The authors acknowledge financial support from ASTAR under SERC grant No. 0521010043. Special thanks to a referee initiating the discussion on the beam waist ratios in this paper.

* christian.kurtsiefer@gmail.com; http://www . quantumlah.org

[1] R. Ghosh and L. Mandel, Phys. Rev. Lett. 59, 1903 (1987); Z. Y. Ou and L. Mandel, Phys. Rev. Lett. 61, 50 (1988), Y. H. Shih and C. O. Alley, Phys. Rev. Lett. 61, 2921 (1988).

[2] W. H. Louisell, A. Yariv and A. E. Siegman, Phys. Rev.
124, 1646 (1961); T. G. Giallorenzi and C. L. Tang, Phys. Rev. 166, 225 (1968); D. C. Burnham and W. L. Weinberg, Phys. Rev. Lett. 25, 84 (1970).

[3] D. A. Kleinman, Phys. Rev. 174, 1027 (1968).

[4] D. N. Klyshko, Photons and Non-linear Optics (Gordon and Breach Science Publishers, 1988).

[5] C. K. Hong and L. Mandel, Phys. Rev. A 31, 2409 (1985).

[6] C. H. Monken, P. H. Souto Ribeiro, and S. Pádua, Phys. Rev. A 57, R2267 (1998).

[7] S. Castellato, I. P. Degiovanni, A. Migdall and M. Ware, N. J. Physics 6, 87 (2004); S. Castellato, I. P. Degiovanni, V. Schettini and A. Migdall, IEEE Trans. Inst. Meas. 54, 890 (2005).

[8] F. A. Bovino, P. Varisco, A. M. Colla, G. Castagnoli, G. D. Giuseppi, and A. V. Sergienko, Opt. Comms. 227, 343 (2003).

[9] D. Ljunggren and M. Tengner, Phys. Rev. A 72, 062301 (2005),

[10] C. Kurtsiefer, M. Oberparleiter, and H. Weinfurter, Phys. Rev. A 64, 023802 (2001).

[11] P. G. Kwiat, K. Mattle, H. Weinfurter, A. Zeilinger, A. V. Sergienko, and Y. Shih, Phys. Rev. Lett. 75, 4337 (1995).

[12] P. G. Kwiat, E. Waks, A. G. White, I. Appelbaum, and P. H. Eberhard, Phys. Rev. A 60, R773 (1999).

[13] Y. R. Shen, The Principles of Nonlinear Optics (Wiley Interscience, 2003).

[14] N. Boeuf, D. Branning, I. Chaperot, E. Dauler, S. Guerin, G. Jaeger, A. Muller, and A. Migdall, Opt. Eng. 39, 1016 (2000).

[15] K. Koch, E. C. Cheung, G. T. Moore, S. H. Chakimakijan, and J. M. Liu, IEEE J. of Quant. Elec. 31, 769 (1995).

[16] M. Fiorentino, S. M. Spillane, R. G. Beausoleil, T. D. Roberts, P. Battle, and M. W. Munro, Opt. Express 15, 7479 (2007).

[17] C. Branciard, A. Ling, N. Gisin, C. Kurtsiefer, A. LamasLinares, and V. Scarani, Phys. Rev. Lett. 99, 210407 (2007).

[18] R. S. Klein, G. E. Kugel, A. Maillard, A. Sifi, and K. Polgar, Optical Materials 22, 163 (2003).

[19] I. Marckic, A. Lamas-Linares, and C. Kurtsiefer, Appl. Phys. Lett. 89, 101122 (2006).

[20] K. Sanaka, K. Kawahara, and T. Kuga, Phys. Rev. Lett. 86, 5620 (2001).

[21] S. Tanzilli et al., Elec. Lett. 37, 26 (2001).

[22] A. B. U'Ren, C. Silberhorn, K. Banaszek, and I. A. Walmsley, Phys. Rev. Lett. 93, 093601 (2004). 